\title{
PACAP27 regulates ciliary function in primary cultures of rat brain ependymal cells
}

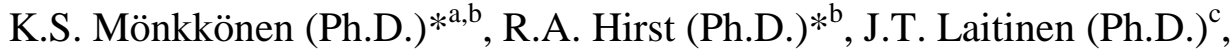 \\ and C. O'Callaghan (MD, Ph.D.) $)^{\mathrm{b}}$
}

Address: ${ }^{a}$ Department of Pharmacology \& Toxicology, University of Kuopio, Kuopio, FIN70211, Finland, ${ }^{b}$ Department of Infection, Immunity and Inflammation, University of Leicester, Leicester LE2 7LX, UK, ${ }^{c}$ Institute of Biomedicine, University of Kuopio, Kuopio, FIN-70211, Finland.

* equal contribution

Correspondence should be addressed to:

Kati S. Mönkkönen

INSERM - UCBL1 U842, Department of Neuro-oncology and Neuroinflammation, Faculté Laënnec, Rue Guillaume Paradin, 69372 LYON, FRANCE

Tel. +3304 78778759, Fax. +330478778616

E-mail Kati.Monkkonen@uku.fi 


\begin{abstract}
Ependymal cells line the brain ventricles and separate the CSF from the underlying neuronal tissue. The function of ependymal cilia is largely unclear however they are reported to be involved in the regulation of CSF homeostasis and host defence against pathogens. Here we present data that implicates a role of pituitary adenylate cyclase-activating polypeptide (PACAP) in the inhibition of ependymal ciliary function, and also that the PACAP effects are not entirely dependent on adenylyl cyclase activation. Primary ependymal cultures were treated with increasing doses of PACAP27 or adenylyl cyclase toxin (ACT), and ciliary beating was recorded using high-speed digital video imaging. Ciliary beat frequency (CBF) and amplitude were determined from the videos. Ependymal CBF and ciliary amplitude were attenuated by PACAP27 in a concentration- and time-dependent manner. The peptide antagonist PACAP6-27 blocked PACAP27-induced decreases in amplitude and CBF. Treatment with ACT caused a decrease in amplitude but had no effect on CBF, this suggests that the inhibition of CBF and amplitude seen with PACAP27 may not be completely explained by $\mathrm{G}_{\mathrm{s}}$-AC-cAMP pathway. We present here the first observational study to show that activation of PAC1 receptors with PACAP27 has an important role to play in the regulation of ependymal ciliary function.
\end{abstract}

Key words: Ependymal cilia, ciliary function, pituitary adenylate cyclase-activating polypeptide, PACAP, PAC1 receptor 


\section{Introduction}

Ependymal cells lining the ventricular cavities form a protective interface between brain parenchyma and the circulating cerebrospinal fluid (CSF). Ependymal cells bear approximately 40 motile cilia per cell which beat in the coordinated pattern at approximately $40 \mathrm{~Hz}$ (Del Bigio, 1995; O'Callaghan et al., 1999) that rapidly move CSF adjacent to the ventricular walls. The precise functional roles of ependymal cilia are not fully understood, however, it is known that abnormal ciliary function (Banizs et al., 2005; Mönkkönen et al., 2007; Shimizu and Koto, 1992), associated with an inherited condition known as Primary Ciliary Dyskinesia (PCD) can result in hydrocephalus (Ibañez-Tallon et al., 2004). Ependymal cilia also have been shown to play an important role in host defence against pathogens (Hirst et al., 2003; Hirst et al., 2000; Mohammed et al., 1999). In addition, a recent study (Sawamoto et al., 2006) has shown that ependymal cilia are responsible for the formation of gradients of CSF guidance molecules that direct neuroblast movement in the brain parenchyma. However, few studies have addressed the question of hormonal regulation of the ciliary function.

There is previous evidence that rat ependymal ciliary function could be inhibited by an excess activity of the adenylyl cyclase (AC) - cAMP pathway (Mönkkönen et al., 2007). The dual balance between $G$ protein families $G_{i}$ and $G_{s}$ in the regulation of $A C$ is well established, but $\mathrm{G}$ protein-coupled receptors have not been identified in CBF inhibition. Interestingly, a recent study showed that mice overexpressing PAC1 receptors developed hydrocephalus with excess activity of PKA pathway (Lang et al., 2006). PAC1 receptors are activated by pituitary adenylate cyclase-activating polypeptide (PACAP) and coupled to $\mathrm{G}_{\mathrm{s}}$ protein family. This 
suggests that endogenous neuropeptide, PACAP, may have a receptor-dependent role in the regulation of ciliary function.

During evolution, PACAP has been remarkably well conserved, and it shares a striking sequence similarity between species (Arimura, 1998) and suggests a vital physiological role. PACAP is a pleiotropic peptide, involved in the regulation of hormone release from the pituitary and adrenal gland. PACAP has been implicated in a variety of biological processes such as reproduction, development, growth, immune responses, cardiovascular, gastrointestinal and respiratory functions (Arimura, 1998; Vaudry et al., 2000). In the CNS, PACAP acts as a neurotrophic factor during brain development and as a neuroprotective factor during adulthood (Arimura, 1998; Vaudry et al., 2000; Watanabe et al., 2007). Further, it is a neurotransmitter and/or a neuromodulator in the PNS and a preganglionic neurotransmitter in the CNS (Arimura, 1998). Physiological concentrations of PACAP in CSF and brain tissue are in the range of 0.1-2 nM (Ohno et al., 2005; Wilderman and Armstead, 1997). In rat brain, high levels of PACAP are mainly found in the hypothalamus. PACAP acts as the transmitter of the monosynaptic neuronal pathway (the retinohypothalamic tract) mediating light information to the master biological clock, the hypothalamic suprachiasmatic nuclei (Hannibal, 2006). In addition, regions adjacent to the ventricles, like septal, paraventricular and periventricular nuclei, have high PACAP concentrations as well as high density of PACAP immunopositive cells and fibers in both rat and human (Arimura et al., 1991; Ghatei et al., 1993; Köves et al., 1991; Masuo et al., 1993; Palkovits et al., 1995; Vigh et al., 1991).

We have previously described a primary rat ependymal culture model that allows ciliary function to be studied over time (Hirst et al., 2000). An often ignored fact regarding the 
ciliary function is that following certain topical toxic insults the $\mathrm{CBF}$ remains unchanged whilst the ciliary amplitude, and hence function, may be disturbed (Chilvers and O'Callaghan, 2000). The present study allowed us to use our methodology to determine the role of PACAP in both ependymal CBF and amplitude.

\section{Materials and methods}

\subsection{Ependymal Cell Culture}

Ciliated rat ependymal cells were grown using a method adapted from Wiebel and colleagues (Weibel et al., 1986) and described previously (Hirst et al., 2000).

\subsection{Compound addition}

All experiments were performed in Hepes-buffered minimum essential media (MEM) without additives. PACAP27 (Tocris, Bristol, UK), PACAP6-27 (Sigma-Aldrich, Poole, UK) and adenylyl cyclase toxin (Sigma-Aldrich) were diluted to their final concentrations in $1 \mathrm{ml}$ MEM. Negative controls were incubated in $1 \mathrm{ml}$ of MEM alone. The compounds were added on the cells at 0 min (all compounds used), 120 min (PACAP27, PACAP27 combined with PACAP6-27, ACT) and $240 \mathrm{~min}$ (PACAP27) in increasing concentrations. In the case of PACAP27 and peptide antagonist PACAP6-27 combination, the cells were pre-treated for 60 min with the peptide antagonist.

\section{3. $C B F$ and amplitude}

To determine CBF and amplitude the cells were placed in a humidified (70-90\%) incubation $\left(37^{\circ} \mathrm{C}\right)$ chamber and observed from above using an inverted microscope system (Diphot, 
Nikon, UK). In order to prevent cilia-to-cilia variation, we selected a long strip of mature cilia for each recording, and followed the function of the same cilia throughout the experiment. From each recording, 5 cilia were randomly selected for $\mathrm{CBF}$ and amplitude analysis. To avoid any influence of potentially varying degree of beat pattern on the results, only the mature cilia which displayed their full beat cycle when viewed from above were included. Beating cilia were recorded using a digital high-speed video camera (Troubleshooter, Lake Image Systems, UK) at a rate of 500 frames per second, using a shutter speed of 1 in 2000. The camera allows video sequences to be recorded and played back at reduced frame rates or frame by frame. $\mathrm{CBF}$ was determined by timing a given number of individual cilia beat cycles. Basal CBF was measured at 0 minutes, before addition of compounds. Calculation of $\mathrm{CBF}(\mathrm{Hz})$ : Frequency $(\mathrm{Hz})=$ Number of frames recorded per second $/$ frames elapsed for 5 ciliary beat cycles X 5 (conversion per beat cycle). Ciliary amplitude was measured using a similar method as previously described (O'Callaghan et al., 2008). The captured video sequences were played back at a slow rate which allowed determination of the distance travelled by the cilia tips within the power stroke of the beat cycle.

\subsection{Data analysis}

Data are presented as mean \pm SEM of CBF and amplitude measurements from 4-11 separate cell cultures. The mean reading of each individual culture was obtained from 5 randomly chosen cilia. Statistical comparisons were made using one-way analysis of variance with Tukey's multiple comparison test and Student's unpaired two-tailed t-test. All analysis was made using GraphPad Prism for Windows (GraphPad Software, Inc., San Diego, CA). Statistical significance was achieved when $\mathrm{p}<0.05$. 


\section{Results}

\subsection{PACAP27 caused a decrease in CBF and amplitude.}

Primary ependymal cultures, which had basal CBF of approximately $40 \mathrm{~Hz}$ and basal amplitude of approximately $9 \mu \mathrm{m}$, were treated with increasing concentrations of PACAP27. At time points indicated by arrows (Figure 1), the media was completely replaced both in control cells (with fresh culture media) and in PACAP27 cells (with media containing $10 \mathrm{nM}$, $100 \mathrm{nM}$ or $1 \mu \mathrm{M}$ of PACAP27). The arrows thus denote the arrival of the agonist at the receptors. Beating cilia were recorded using a digital high-speed video camera at $30 \mathrm{~min}$ intervals in PACAP27 treated and at 60 min intervals in control cells.

Our data showed that PACAP27 caused a decrease in CBF (Figure 1A) and amplitude (Figure 1B), and that this decrease was statistically significant $\left({ }^{*} \mathrm{p}<0.05, * * * \mathrm{p}<0.001\right.$; Student's unpaired $t$ test). This effect was both concentration- and time-dependent. After addition of 10 $\mathrm{nM}, 100 \mathrm{nM}$ and $1 \mu \mathrm{M}$ PACAP27, CBF was approximately $93 \%, 86 \%$ and $73 \%$ of the basal CBF, while average amplitude was $83 \%, 60 \%$ and $37 \%$ of the basal amplitude, respectively. Based on these data, we concluded that PACAP27 effects on CBF and amplitude were linear in the concentration range used. In the control cells, $\mathrm{CBF}$ and amplitude remained at the baseline level (approximately $35 \mathrm{~Hz}$ and $7 \mu \mathrm{m}$ ) during the whole experiment. 24 hours after PACAP27 was removed from the ependymal cells by media replacement, the inhibition of CBF and amplitude was reversed (data not shown).

3.2. PACAP27 mediated decrease in CBF and amplitude was inhibited by the competitive peptide antagonist PACAP6-27. 
In order to clarify whether the PACAP27 effects on $\mathrm{CBF}$ and amplitude were receptormediated, we used $3 \mu \mathrm{M}$ concentration of the peptide antagonist PACAP6-27 in combination with $1 \mu \mathrm{M}$ PACAP27 (Figure 2). The baseline CBF and amplitude levels in control cells, PACAP27 cells and in cells treated with combination of PACAP27 and the antagonist PACAP6-27, were $34 \mathrm{~Hz}, 38 \mathrm{~Hz}$ and $44 \mathrm{~Hz}$, respectively. The corresponding baseline amplitude levels were $6 \mu \mathrm{m}, 8 \mu \mathrm{m}$ and $8 \mu \mathrm{m}$. Compounds were added on cells at the time points indicated by arrows.

Figure 2 shows that PACAP27 addition inhibited both CBF and amplitude and this effect was negated by pre-incubation with the antagonist. At the 120 minutes time point mean $\mathrm{CBF}$ (Figure 2A) and mean amplitude (Figure 2B) of the PACAP27 treated ependymal cells were significantly different from the control (* $\mathrm{p}<0.05$; One-way ANOVA with Tukey's Multiple Comparison test). In the control cells and in cells treated with combination of PACAP27 and PACAP6-27, the CBF and amplitude remained at their baseline levels throughout the whole experiment. $3 \mu \mathrm{M}$ PACAP6-27 administered alone did not change CBF or amplitude (data not shown).

\subsection{Adenylyl cyclase toxin caused a decrease in ciliary amplitude but not CBF.}

In order to simulate the intracellular rise in cAMP following receptor stimulation by PACAP27, we used ACT treatment on the ependymal cells (Figure 3). ACT containing media was added on cells in increasing concentrations of $10 \mathrm{ng} / \mathrm{ml}$ and $30 \mathrm{ng} / \mathrm{ml}$ at time points indicated by arrows. Media was replaced on control cells at the same time points. Baseline CBF was approximately $40 \mathrm{~Hz}$ and amplitude $8 \mu \mathrm{m}$. 
ACT caused a statistically significant decrease in the ciliary amplitude (Figure $3 \mathrm{~B}, * * \mathrm{p}<0.01$; Student's unpaired $t$ test) that was similar to the action of PACAP27. However, during the 240 min experiment it did not significantly change CBF (Figure 3A). CBF and amplitude in the control cells remained at their baseline levels of approximately $35 \mathrm{~Hz}$ and $7 \mu \mathrm{m}$ during the whole experiment.

\section{Discussion}

This study aimed to clarify whether PACAP has a role in controlling ependymal ciliary function. Our results showed that PACAP27 reduced CBF and amplitude in cultured rat ependymal cilia in a receptor-mediated manner. Furthermore, our results suggest that the effects of PACAP27 may not be completely explained by effect on the AC-cAMP pathway. Increasing the cAMP using low dose ACT treatment inhibited ependymal ciliary amplitude but did not affect the CBF over the course of the study.

The role of ependymal ciliary beating and the control of CSF directional flow has been well described by others (Cathcart and Worthington, 1964; Yamadori and Nara, 1979). However, a recent study showed that ependymal ciliary beating was crucial for the formation of concentration gradients of CSF guidance molecules directing proper neuroblast migration (Sawamoto et al., 2006). The connection between ciliary function and CSF homeostasis has been clearly demonstrated, as ciliary dysfunction or immotility (Nakamura and Sato, 1993; Shimizu and Koto, 1992) as well as defects in ciliary structure components (Ibañez-Tallon et al., 2002; Ibañez-Tallon et al., 2004; Sapiro et al., 2002) or defects in proteins involved in ciliogenesis (Chen et al., 1998; Kobayashi et al., 2002; Taulman et al., 2001) may all result in 
hydrocephalus. Further, in patients with PCD, there are reports of associated enlarged ventricles and hydrocephalus (Ibañez-Tallon et al., 2002). All of the cilia of PCD patients are either immotile or beat in a dyskinetic fashion (Chodhari et al., 2004). Recently, the connection between dysfunctional cilia and the development of hydrocephalus was clarified further, as it was demonstrated, that severely malformed and dysfunctional cilia in choroid plexus in vivo results in disturbed cAMP-regulated chloride transport, leading to excess CSF production and hydrocephalus (Banizs et al., 2005).

There is evidence of the involvement of AC-cAMP pathway in inhibition of ependymal ciliary function. Our previous study showed that attenuation of the inhibitory G protein subunit $\mathrm{G}_{\mathrm{i} 2}$ resulted in ventricular dilatation and ciliary stasis in vitro (Mönkkönen et al., 2007). This can be explained by a disturbed balance of AC regulation by $\mathrm{G}_{\mathrm{s}}-\mathrm{G}_{\mathrm{i}}$. The present study has confirmed the involvement of cAMP in the control of ciliary function, as ACT was shown to significantly decrease ciliary amplitude. In line with these findings, mice overexpressing PAC1 receptor have been shown to have elevated PKA and PKC activity, ciliary defects and increased apoptosis, resulting in development of hydrocephalus (Lang et al., 2006).

While there are yet few reports on regulation of ciliary beat in the brain, tracheal ciliary function has been a focus of many studies. Although many structural and functional features seems well conserved between different ciliated tissues (Satir and Christensen, 2007), the ciliary beat-regulating pathways seems not to be shared. In contrast, ciliary beat regulatory molecules may cause completely reverse effects on the CBF in different tissues. Activation of the PKA pathway by cAMP or ATP increases tracheal CBF (Di Benedetto et al., 1991; Morse et al., 2001), whereas the same pathway is associated with CBF inhibition in the ependymal 
cilia (Mönkkönen et al., 2007). In general, outer dynein arms regulate the power stroke and thus the $\mathrm{CBF}$, and inner dynein arms regulate bending of the cilium (Brokaw and Kamiya, 1987; Habermacher and Sale, 1997; Satir et al., 1995). In tracheal cilia, the underlying mechanism behind the $\mathrm{CBF}$ increase has been proposed to be cAMP-dependent activation of axonemal PKA, which causes dynein phosphorylation (Salathe, 2007). Studies on Tetrahymena have shown that the ciliary amplitude changes as a result of direct $\mathrm{Ca}^{2+}$ binding to inner dynein arms (Guerra et al., 2003). Potentially, the amplitude decrease that we observed in the present study by PACAP27 and ACT could be explained by altered inner dynein arm activity, evoked by a cAMP-dependent pathway.

PACAP exists in two forms, PACAP38 and PACAP27 (Miyata et al., 1989; Miyata et al., 1990). Both originate from a single gene and have a common 175-176-amino acid precursor (Arimura, 1998; Arimura and Shioda, 1995). Although PACAP38 is the predominant form found in tissues, the N-terminal amino acids 1-27, which correspond to PACAP27, account for biological activity (Arimura and Shioda, 1995; Vaudry et al., 2000). PACAP functions are mediated through three G protein-coupled receptors, PAC1, VPAC1 and VPAC2. While only PACAP has high affinity to PAC1 receptor, the latter two can be activated by vasoactive intestinal peptide (VIP) and PACAP with equal affinity (Harmar et al., 1998). PAC1 has at least eight splice variants, which differ in their cell or tissue expression, and in their ability to activate the alternative signalling pathway, phospholipase C (PLC). VPAC1 and VPAC2 and seven of the PAC1 variants couple to $G_{s}$ family and stimulate AC. One PAC1 variant activates solely L-type $\mathrm{Ca}^{2+}$ channels (Chatterjee et al., 1996; Pantaloni et al., 1996; Spengler et al., 1993). 
The two PACAP forms PACAP27 and PACAP38 are equipotent in stimulation of the $\mathrm{G}_{\mathrm{s}}-\mathrm{AC}$ cAMP pathway, but only PACAP38 can potently stimulate PLC via PAC1 activation (Basille et al., 1995; Spengler et al., 1993). In the present study, we selected PACAP27 in order to clarify the AC-stimulating effects of PACAP in the regulation of ciliary function. PACAP27 has a high affinity to PAC1 and VPAC1, and slightly lower affinity to VPAC2 receptors. Inactive peptide PACAP6-27 acts as antagonist on PAC1 and VPAC2, but not on VPAC1. Since our results showed that PACAP27-induced inhibitory effects on ciliary function were fully inhibited by PACAP6-27, VPAC1 receptor seems not to have a role in mediating the observed ciliary inhibition.

Concerning the receptor(s) mediating the inhibitory effects of PACAP27 on CBF and amplitude, the earlier study on PAC1 overexpressing mice strongly supports involvement of PAC1 receptors. Namely, PAC1 overexpressing mice showed a higher level of PAC1 expression especially in ventricle-surrounding regions, when compared to wild type (Lang et al., 2006). In the developing brain, PAC1 mRNA expression showed more intense subventricular localization (Basille et al., 2000; Jaworski and Proctor, 2000; Zhou et al., 1999) than in the adult (Hashimoto et al., 1996; Jaworski and Proctor, 2000; Shioda et al., 1997). There is evidence that PAC1 mRNA intensity in ventricular regions remains high during the first postnatal week (Jaworski and Proctor, 2000), which corresponds to the age of the ependymal cell cultures used in the present study. Unlike PAC1, VPAC2 is not present in subventricular regions during brain development (Basille et al., 2000), and in situ hybridisation studies have not provided evidence of its presence in the rat ventricle and surrounding regions (Basille et al., 2000; Sheward et al., 1995; Usdin et al., 1994). This suggests that PAC1 receptor might account for PACAP-mediated regulation of ciliary function. 
Interestingly, while classical intracellular responses to $\mathrm{G}$ protein-coupled receptor activation are expected to be rapid, the regulatory effects of PACAP27 on ependymal ciliary function observed in the present study emerged with time and were progressive. The observed delay strongly suggests that ependymal ciliary function might be regulated in a more complicated manner than via simple receptor activation. Potentially, regulation might involve changes in the energy metabolism, or participation of the various PAC1 isoforms, which could mediate the signal further into different downstream pathways.

Regarding the role of neuropeptide PACAP in CSF homeostasis, patients with gene multiplications at regions where PACAP and PAC1 genes reside (18p11 and 7p15, respectively) (Hosoya et al., 1992; Ogi et al., 1993), have been reported to develop hydrocephalus (Miller et al., 1979; Takeda et al., 1989). Further, PAC1 overexpression in mice results in hydrocephalus (Lang et al., 2006). Consistent with these findings, we have shown for the first time, a receptor-dependent regulation of ciliary beat pattern by PACAP27.

\section{Statement of conflict of interest}

None

\section{Acknowledgements}

We wish to thank Mr Norman Baker for skilful technical assistance. During this study KSM was financially supported by The Association of Finnish Pharmacies, Finnish Pharmaceutical Society, Alfred Kordelin Foundation, Farmos Science and Research Foundation, Helsingin Sanomat Centennial Foundation and The Saastamoinen Foundation. 


\section{References}

Arimura, A., 1998. Perspectives on pituitary adenylate cyclase activating polypeptide (PACAP) in the neuroendocrine, endocrine, and nervous systems. Japanese Journal of Physiology. 48, 301-31.

Arimura, A., Shioda, S., 1995. Pituitary adenylate cyclase activating polypeptide (PACAP) and its receptors: neuroendocrine and endocrine interaction. Frontiers in Neuroendocrinology. 16, 53-88.

Arimura, A., et al., 1991. Tissue distribution of PACAP as determined by RIA: highly abundant in the rat brain and testes. Endocrinology. 129, 2787-9.

Banizs, B., et al., 2005. Dysfunctional cilia lead to altered ependyma and choroid plexus function, and result in the formation of hydrocephalus. Development. 132, 5329-39.

Basille, M., et al., 1995. Pituitary adenylate cyclase-activating polypeptide (PACAP) stimulates adenylyl cyclase and phospholipase $\mathrm{C}$ activity in rat cerebellar neuroblasts. Journal of Neurochemistry. 65, 1318-24.

Basille, M., et al., 2000. Comparative distribution of pituitary adenylate cyclase-activating polypeptide (PACAP) binding sites and PACAP receptor mRNAs in the rat brain during development. Journal of Comparative Neurology. 425, 495-509.

Brokaw, C. J., Kamiya, R., 1987. Bending patterns of Chlamydomonas flagella: IV. Mutants with defects in inner and outer dynein arms indicate differences in dynein arm function. Cell Motility and the Cytoskeleton. 8, 68-75.

Cathcart, R. S., 3rd, Worthington, W. C., Jr., 1964. Ciliary Movement In The Rat Cerebral Ventricles: Clearing Action And Directions Of Currents. Journal of Neuropathology and Experimental Neurology. 23, 609-18.

Chatterjee, T. K., et al., 1996. Molecular cloning of a novel variant of the pituitary adenylate cyclase-activating polypeptide (PACAP) receptor that stimulates calcium influx by activation of L-type calcium channels. Journal of Biological Chemistry. 271, 3222632.

Chen, J., et al., 1998. Mutation of the mouse hepatocyte nuclear factor/forkhead homologue 4 gene results in an absence of cilia and random left-right asymmetry. Journal of Clinical Investigation. 102, 1077-82.

Chilvers, M. A., O'Callaghan, C., 2000. Analysis of ciliary beat pattern and beat frequency using digital high speed imaging: comparison with the photomultiplier and photodiode methods. Thorax. 55, 314-7.

Chodhari, R., et al., 2004. Cilia, primary ciliary dyskinesia and molecular genetics. Paediatric Respiratory Reviews. 5, 69-76.

Del Bigio, M. R., 1995. The ependyma: a protective barrier between brain and cerebrospinal fluid. Glia. 14, 1-13.

Di Benedetto, G., et al., 1991. Effect of cyclic AMP on ciliary activity of human respiratory epithelium. European Respiratory Journal. 4, 789-95.

Ghatei, M. A., et al., 1993. Distribution, molecular characterization of pituitary adenylate cyclase-activating polypeptide and its precursor encoding messenger RNA in human and rat tissues. Journal of Endocrinology. 136, 159-66.

Guerra, C., et al., 2003. Cloning, localization, and axonemal function of Tetrahymena centrin. Molecular Biology of the Cell. 14, 251-61.

Habermacher, G., Sale, W. S., 1997. Regulation of flagellar dynein by phosphorylation of a 138-kD inner arm dynein intermediate chain. Journal of Cell Biology. 136, 167-76.

Hannibal, J., 2006. Roles of PACAP-containing retinal ganglion cells in circadian timing. International Review of Cytology. 251, 1-39. 
Harmar, A. J., et al., 1998. International Union of Pharmacology. XVIII. Nomenclature of receptors for vasoactive intestinal peptide and pituitary adenylate cyclase-activating polypeptide. Pharmacological Reviews. 50, 265-70.

Hashimoto, H., et al., 1996. Distribution of the mRNA for a pituitary adenylate cyclaseactivating polypeptide receptor in the rat brain: an in situ hybridization study. Journal of Comparative Neurology. 371, 567-77.

Hirst, R. A., et al., 2003. Streptococcus pneumoniae damages the ciliated ependyma of the brain during meningitis. Infection and Immunity. 71, 6095-100.

Hirst, R. A., et al., 2000. Effect of pneumolysin on rat brain ciliary function: comparison of brain slices with cultured ependymal cells. Pediatric Research. 47, 381-4.

Hosoya, M., et al., 1992. Structure of the human pituitary adenylate cyclase activating polypeptide (PACAP) gene. Biochimica et Biophysica Acta. 1129, 199-206.

Ibañez-Tallon, I., et al., 2002. Loss of function of axonemal dynein Mdnah5 causes primary ciliary dyskinesia and hydrocephalus. Human Molecular Genetics. 11, 715-21.

Ibañez-Tallon, I., et al., 2004. Dysfunction of axonemal dynein heavy chain Mdnah5 inhibits ependymal flow and reveals a novel mechanism for hydrocephalus formation. Human Molecular Genetics. 13, 2133-41.

Jaworski, D. M., Proctor, M. D., 2000. Developmental regulation of pituitary adenylate cyclase-activating polypeptide and PAC(1) receptor mRNA expression in the rat central nervous system. Brain Research. Developmental Brain Research. 120, 27-39.

Kobayashi, Y., et al., 2002. Hydrocephalus, situs inversus, chronic sinusitis, and male infertility in DNA polymerase lambda-deficient mice: possible implication for the pathogenesis of immotile cilia syndrome. Molecular and Cellular Biology. 22, 276976.

Köves, K., et al., 1991. Comparative distribution of immunoreactive pituitary adenylate cyclase activating polypeptide and vasoactive intestinal polypeptide in rat forebrain. Neuroendocrinology. 54, 159-69.

Lang, B., et al., 2006. Expression of the human PAC1 receptor leads to dose-dependent hydrocephalus-related abnormalities in mice. Journal of Clinical Investigation. 116, 1924-34.

Masuo, Y., et al., 1993. Regional distribution of pituitary adenylate cyclase activating polypeptide (PACAP) in the rat central nervous system as determined by sandwichenzyme immunoassay. Brain Research. 602, 57-63.

Miller, M., et al., 1979. Familial, balanced insertional translocation of chromosome 7 leading to offspring with deletion and duplication of the inserted segment, $7 \mathrm{p} 15$ leads to $7 \mathrm{p} 21$. American Journal of Medical Genetics. 4, 323-32.

Miyata, A., et al., 1989. Isolation of a novel 38 residue-hypothalamic polypeptide which stimulates adenylate cyclase in pituitary cells. Biochemical and Biophysical Research Communications. 164, 567-74.

Miyata, A., et al., 1990. Isolation of a neuropeptide corresponding to the N-terminal 27 residues of the pituitary adenylate cyclase activating polypeptide with 38 residues (PACAP38). Biochemical and Biophysical Research Communications. 170, 643-8.

Mohammed, B. J., et al., 1999. The effect of the pneumococcal toxin, pneumolysin on brain ependymal cilia. Microbial Pathogenesis. 27, 303-9.

Mönkkönen, K. S., et al., 2007. Intracerebroventricular antisense knockdown of G alpha i2 results in ciliary stasis and ventricular dilatation in the rat. BMC Neuroscience. 8, 26.

Morse, D. M., et al., 2001. Differential effects of UTP, ATP, and adenosine on ciliary activity of human nasal epithelial cells. American Journal of Physiology. Cell Physiology. 280, C1485-97. 
Nakamura, Y., Sato, K., 1993. Role of disturbance of ependymal ciliary movement in development of hydrocephalus in rats. Childs Nervous System. 9, 65-71.

O'Callaghan, C., et al., 1999. Respiratory and brain ependymal ciliary function. Pediatric Research. 46, 704-7.

O'Callaghan, C. L., et al., 2008. The effect of viscous loading on brain ependymal cilia. Neuroscience Letters. 439, 56-60.

Ogi, K., et al., 1993. Molecular cloning and functional expression of a cDNA encoding a human pituitary adenylate cyclase activating polypeptide receptor. Biochemical and Biophysical Research Communications. 196, 1511-21.

Ohno, F., et al., 2005. Pituitary adenylate cyclase-activating polypeptide promotes differentiation of mouse neural stem cells into astrocytes. Regulatory Peptides. 126, $115-22$.

Palkovits, M., et al., 1995. Concentrations of pituitary adenylate cyclase activating polypeptide (PACAP) in human brain nuclei. Brain Research. 699, 116-20.

Pantaloni, C., et al., 1996. Alternative splicing in the N-terminal extracellular domain of the pituitary adenylate cyclase-activating polypeptide (PACAP) receptor modulates receptor selectivity and relative potencies of PACAP-27 and PACAP-38 in phospholipase C activation. Journal of Biological Chemistry. 271, 22146-51.

Salathe, M., 2007. Regulation of mammalian ciliary beating. Annual Review of Physiology. 69, 401-22.

Sapiro, R., et al., 2002. Male infertility, impaired sperm motility, and hydrocephalus in mice deficient in sperm-associated antigen 6. Molecular and Cellular Biology. 22, 6298305.

Satir, P., et al., 1995. Ciliary beat frequency is controlled by a dynein light chain phosphorylation. Biophysical Journal. 68, 222S.

Satir, P., Christensen, S. T., 2007. Overview of structure and function of mammalian cilia. Annual Review of Physiology. 69, 377-400.

Sawamoto, K., et al., 2006. New neurons follow the flow of cerebrospinal fluid in the adult brain. Science. 311, 629-32.

Sheward, W. J., et al., 1995. The distribution of vasoactive intestinal peptide2 receptor messenger RNA in the rat brain and pituitary gland as assessed by in situ hybridization. Neuroscience. 67, 409-18.

Shimizu, A., Koto, M., 1992. Ultrastructure and movement of the ependymal and tracheal cilia in congenitally hydrocephalic WIC-Hyd rats. Childs Nervous System. 8, 25-32.

Shioda, S., et al., 1997. Localization and gene expression of the receptor for pituitary adenylate cyclase-activating polypeptide in the rat brain. Neuroscience Research. 28, 345-54.

Spengler, D., et al., 1993. Differential signal transduction by five splice variants of the PACAP receptor. Nature. 365, 170-5.

Takeda, K., et al., 1989. Sibs with tetrasomy $18 p$ born to a mother with trisomy $18 p$. Journal of Medical Genetics. 26, 195-7.

Taulman, P. D., et al., 2001. Polaris, a protein involved in left-right axis patterning, localizes to basal bodies and cilia. Molecular Biology of the Cell. 12, 589-99.

Usdin, T. B., et al., 1994. Two receptors for vasoactive intestinal polypeptide with similar specificity and complementary distributions. Endocrinology. 135, 2662-80.

Vaudry, D., et al., 2000. Pituitary adenylate cyclase-activating polypeptide and its receptors: from structure to functions. Pharmacological Reviews. 52, 269-324.

Vigh, S., et al., 1991. Immunohistochemical localization of the neuropeptide, pituitary adenylate cyclase activating polypeptide (PACAP), in human and primate hypothalamus. Peptides. 12, 313-8. 
Watanabe, J., et al., 2007. Localization, characterization and function of pituitary adenylate cyclase-activating polypeptide during brain development. Peptides. 28, 1713-9.

Weibel, M., et al., 1986. Primary culture of rat ependymal cells in serum-free defined medium. Brain Research. 390, 199-209.

Wilderman, M. J., Armstead, W. M., 1997. Role of PACAP in the relationship between cAMP and opioids in hypoxia-induced pial artery vasodilation. American Journal of Physiology. 272, H1350-8.

Yamadori, T., Nara, K., 1979. The directions of ciliary beat on the wall of the lateral ventricle and the currents of the cerebrospinal fluid in the brain ventricles. Scanning Electron Microscopy. 335-40.

Zhou, C. J., et al., 1999. Pituitary adenylate cyclase-activating polypeptide receptors during development: expression in the rat embryo at primitive streak stage. Neuroscience. 93, 375-91. 


\section{Figures and figure legends}
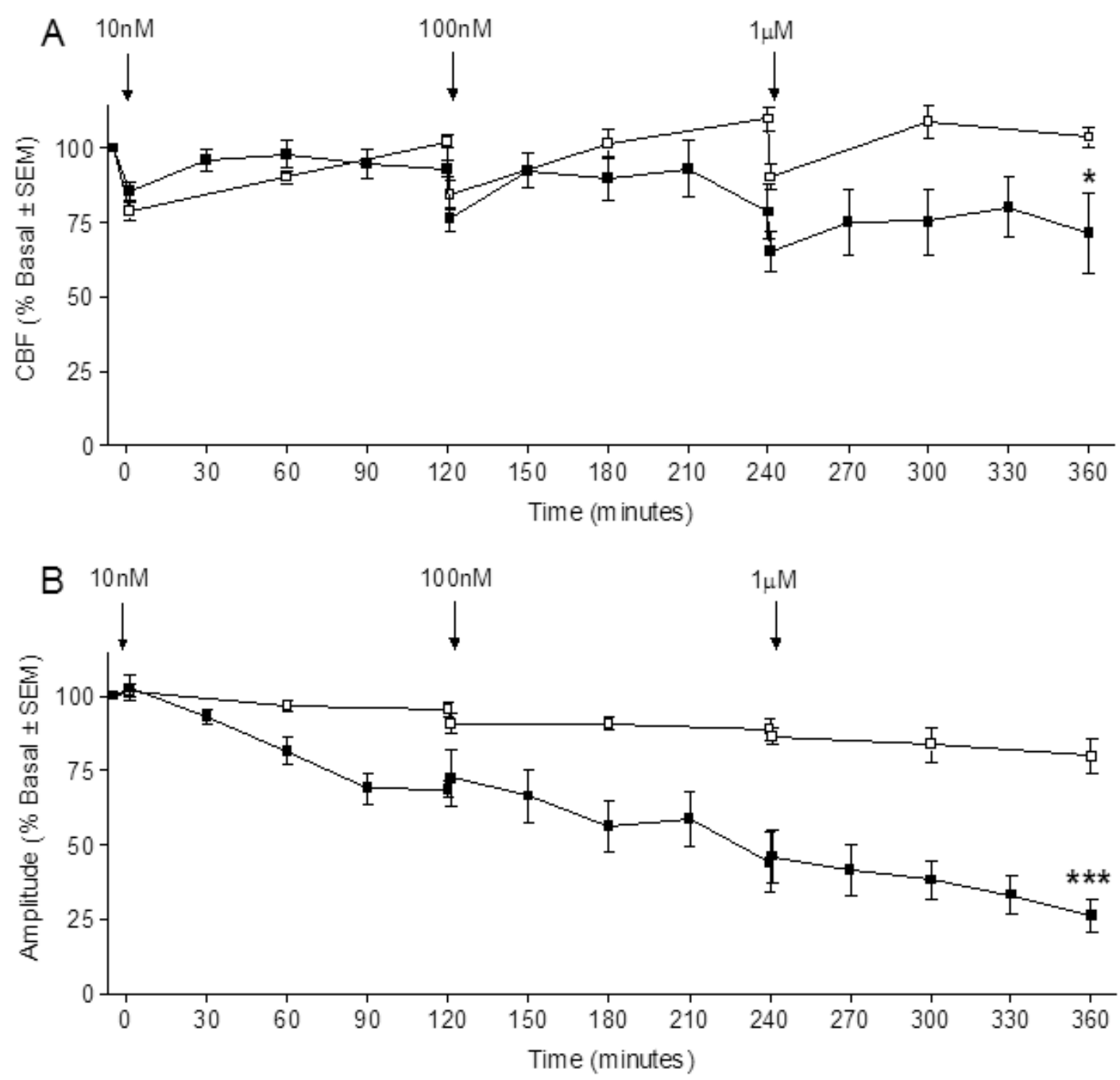

Figure 1. The effect of increasing concentrations of PACAP27 with time on CBF (A) and ciliary beat amplitude (B) in rat ependymal cilia. PACAP27 (ロ) caused a significant decrease in both $\mathrm{CBF}(\mathrm{A})$ and ciliary beat amplitude (B) compared to control (A and B $\square$ ). Arrows show time points of PACAP27 addition in increasing concentrations $(10 \mathrm{nM}, 100 \mathrm{nM}$ and 1 $\mu \mathrm{M})$ or where media was replaced in the controls. CBF and ciliary beat amplitude in the PACAP27 treatment group were significantly different $(* \mathrm{p}<0.05$, *** $\mathrm{p}<0.001$; Student's unpaired $t$ test) from the control. 

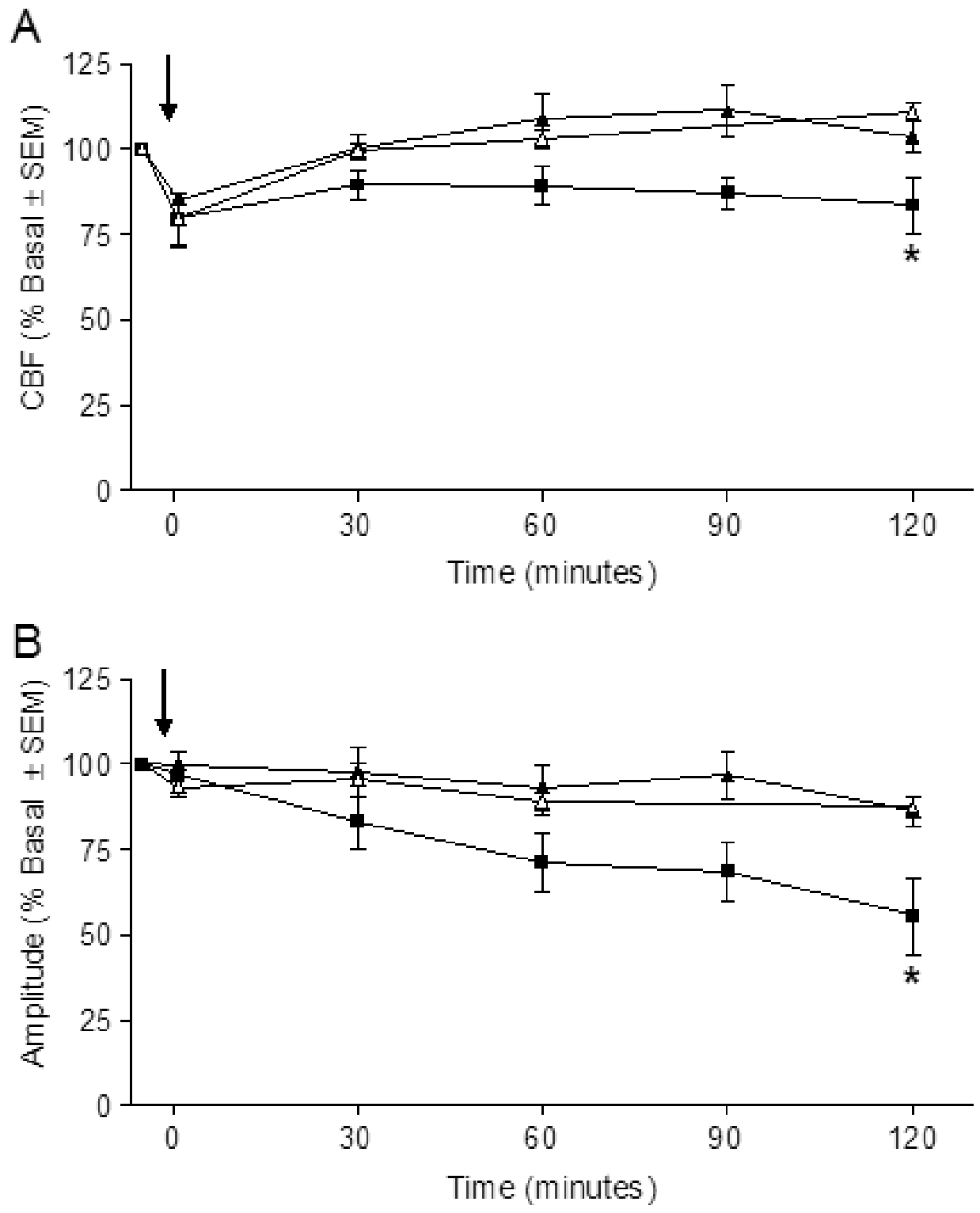

Figure 2. The effect of $1 \mu \mathrm{M}$ PACAP27 alone ( $\mathbf{m})$ and in combination with $3 \mu \mathrm{M}$ peptide antagonist PACAP6-27 ( $\mathbf{\Delta}$ ) on CBF (A) and ciliary beat amplitude (B) in rat ependymal cilia. The PACAP27 effects on amplitude were inhibited by receptor blockade with the antagonist PACAP6-27. Arrows show time points of PACAP27 additions and replacement of control media. At the 120 minutes time point mean amplitude and mean CBF of the PACAP27 treated ependymal cells were significantly (* $\mathrm{p}<0.05$; One-way ANOVA with Tukey’s Multiple Comparison test) different from the control $(\Delta)$. 

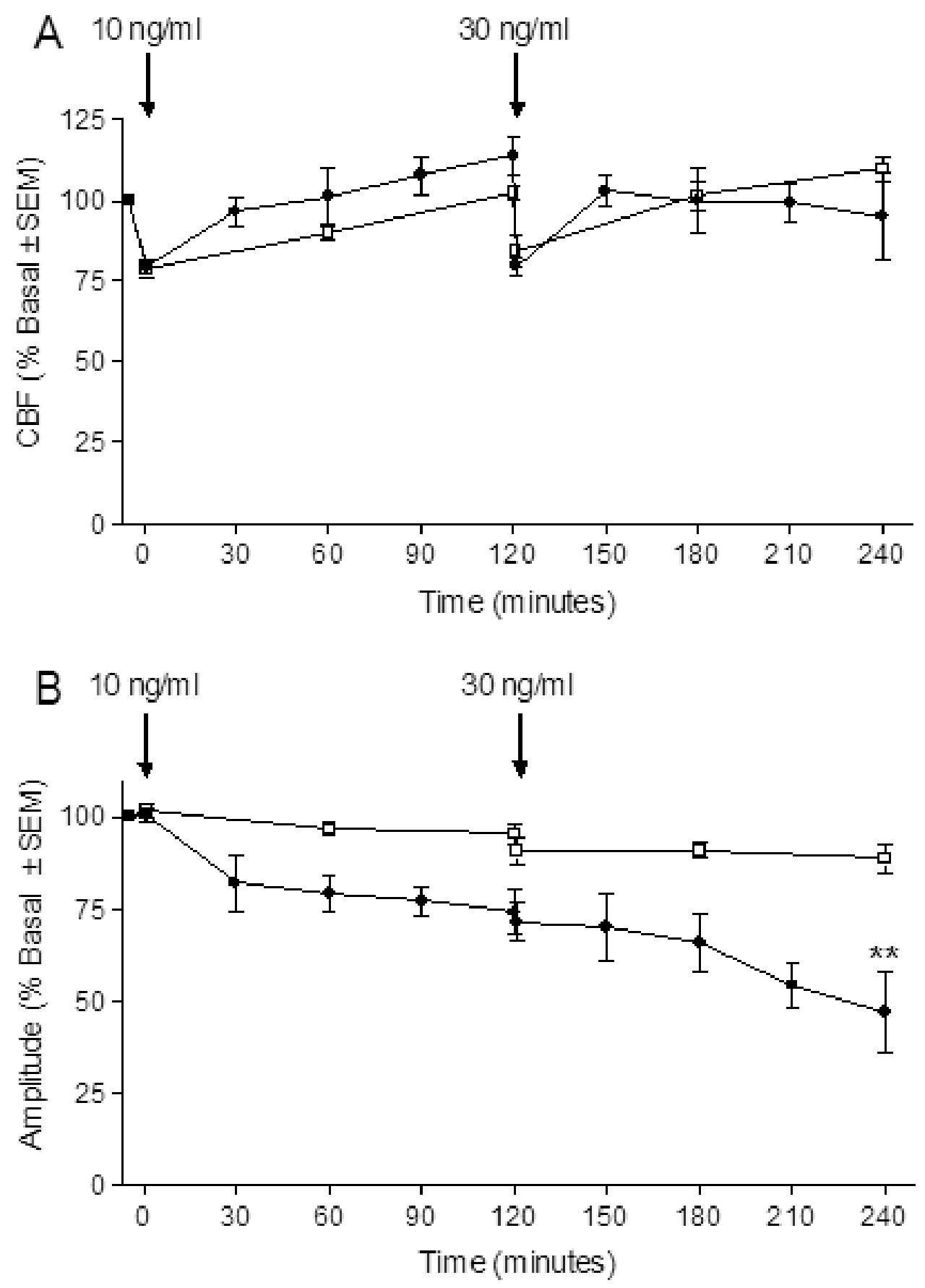

Figure 3. The effect of adenylyl cyclase toxin (ACT) on CBF (A) and ciliary beat amplitude (B) in rat ependymal cilia. ACT $(\bullet)$ caused a significant $(* * \mathrm{p}<0.01$; Student's unpaired t test) decrease in ciliary beat amplitude in rat ependymal cells compared with control ( $\square$ ). Arrows show the time points of ACT additions in increasing concentrations $(10 \mathrm{ng} / \mathrm{ml}$ and $30 \mathrm{ng} / \mathrm{ml})$ or the time of media replacement in the controls. 